\title{
Formación de la competencia de autonomía emocional y moral en estudiantes de turismo.
}

Formation of the competence of emotional and moral autonomy in students of tourism.

MSc. Yulima Valdés Bencomo. ${ }^{1}$, Dr.C Yasser Vázquez Alfonso. ${ }^{2}$ \& Efraín Velasteguí López. ${ }^{3}$

Recibido: 15-03-2017 / Revisado: 20-05-2017 Aceptado: 21-06-2018/ Publicado: 01-07-2018

\begin{abstract}
.
DOI: https://doi.org/10.33262/cienciadigital.v2i3.147

The present investigation covers the results of the theoretical methodological analysis of the authors on the formation of the competence of emotional and moral autonomy from their sustenance in the development teaching based on the Cultural Historical Approach of LS Vygotsky and the diagnosis of the current state in the career of Tourism of the University of Havana. The approach to the category revealed the need for its intentional training to face the emerging challenges of scientific and technological multicultural development, with emphasis on the potential of integral personal development, made it possible to identify the integration of the structural and functional components of the competence, starting of the expression of indicators of the affective cognitive unit in the performance of students in professional situations, which transcend their personal, social and professional performance projection.
\end{abstract}

Keywords: Training, Competence, Emotional Autonomy, Tourism.

\section{Resumen.}

La presente investigación abarca los resultados del análisis teórico metodológico de los autores sobre la formación de la competencia de autonomía emocional y moral desde su sustento en la enseñanza desarrolladora fundamentada en el Enfoque Histórico Cultural de L. S. Vigotsky y el diagnóstico del estado actual en la carrera de

\footnotetext{
${ }^{1}$ Universidad de la Habana, Habana, Cuba, ybencomo@ftur.uh.cu

${ }^{2}$ Universidad de la Habana, Habana, Cuba, yalfos1@gamil.com

${ }^{3}$ Universidad Técnica de Cotopaxi, Ext. La Maná, Cotopaxi, Ecuador, luis.velastegui7838@utc.edu.ec
} 
Turismo de la Universidad de La Habana. La aproximación a la categoría develó la necesidad de su formación intencionada para afrontar los retos emergentes del desarrollo científico tecnológico y multicultural, con énfasis en las potencialidades del desarrollo personal integral, posibilitó identificar la integración de los componentes estructurales y funcionales de la competencia, a partir de la expresión de indicadores de la unidad cognitiva afectiva en la actuación de los estudiantes ante situaciones profesionales, que trascienden a su proyección personal, social y del desempeño profesional.

Palabras Claves: Formación, Competencia, Autonomía Emocional, Turismo.

\section{Introducción .}

El desarrollo acelerado de la ciencia y la tecnología, atribuye hoy a las universidades la responsabilidad de formar profesionales integrales, capaces de dar respuesta a los nuevos cambios, económicos, sociales y políticos generados en los diferentes países de los que son parte.

En Cuba, González (2016), tiene en cuenta varios criterios referidos a la formación como son: enfatizar en la coincidencia de varios autores para definir la formación como un proceso pedagógico y educativo, de mayor o menor complejidad, que sea concebida como la acción sobre un sujeto, partiendo de conocer el fin que se le atribuye, las premisas, las relaciones con otras categorías pedagógicas, las dimensiones, los niveles y las tendencias.

La formación como proceso complejo, conlleva al acercamiento de estudios nuevos, para facilitar la integración de lo estructural y lo funcional. Múltiples han sido las miradas entorno a la formación profesional de los estudiantes, a partir de las responsabilidades que se le asignan a las universidades y del vínculo universidad sociedad, al respecto Hué (2013) destaca dos posiciones, la que desde una visión restringida de sociedad acentúa la preparación de la fuerza laboral, el progreso económico y social que se sustenta en la globalización, y la que posee una visión más amplia de sociedad, que enfatiza en el desarrollo humano (la convivencia multicultural, la práctica profesional con valores éticos, humanistas, de solidaridad).

Según la OEI (2010) la formación profesional se aprecia como el conjunto de procesos de enseñanza aprendizaje de carácter continuo y permanente integrados por acciones técnicopedagógicas destinadas a proporcionar a las personas oportunidades de crecimiento personal, laboral y comunitario brindándoles educación y capacitación socio-laboral al estudiante.

Para los autores del presente artículo, se asume el concepto declarado por el Ministerio de Educación Superior cubano, el cual define la formación de los profesionales como "el proceso que, de modo consciente y sobre bases científicas, se desarrolla en las instituciones de educación superior para garantizar la preparación integral de los estudiantes 
universitarios, que se concreta en una sólida formación científica, técnica y humanista de altos valores ideológicos, políticos, éticos y estéticos, con el fin de lograr profesionales revolucionarios, cultos, competentes, independientes, creadores, para que puedan desempeñarse exitosamente en los diversos sectores de la economía y de la sociedad en general" (MES, 2007: 41).

La formación profesional desde un proceso de enseñanza-aprendizaje desarrollador, se concreta en la adquisición de las competencias en correspondencia con las exigencias de la actividad profesional, es por eso que el propósito de esta investigación fue determinar los fundamentos teórico-metodológicos que sustentan la competencia de autonomía emocional y moral desde la formación del profesional.

\section{La Formación de Competencias Profesionales.}

En nuestros días, se viene tratando el término de competencia a partir de los años 80 del pasado siglo, siempre ha estado asociado a las características psicológicas que posibilitan un desempeño superior. El mismo tiene antecedentes en países como Inglaterra, Estados Unidos, Alemania y Australia.

Su origen etimológico está en el verbo latino "competere", que significa "ir al encuentro una cosa de otra, encontrarse, capacidad para concurrir, coincidir en la dirección" y ha pasado también a las acepciones de: "responder a, corresponder, estar en buen estado, ser suficiente". En sí quiere decir "poder seguir el paso", por lo que una competencia consistiría en la capacidad de seguir en un área determinada; supone una situación de comparación directa y situada en un momento determinado (García y Tobón, 2006).

El término competencia tienen un carácter polisémico y se ha relacionado con sentidos intercambiables y adaptables a las diferentes situaciones y contextos socio-laborales e intenciones comunicativas de los hablantes (Tobón, 2005).

Pinto (1999) declara que cada competencia es entendida como la integración de tres tipos de saberes: conceptual (saber), procedimental (saber hacer) y actitudinal (ser). Según Bisquerra (2004), este término hace alusión a conocimientos, habilidades y actitudes necesarias para realizar actividades diversas con un cierto nivel de calidad y eficacia, donde se integra el saber, saber hacer y saber ser, lo que coincide con la concepción de Pinto (1999).

En este sentido Gonczi y Athanasou (1996) conciben las competencias como una compleja estructura de atributos (conocimientos, actitudes, valores y habilidades) necesarios para el desempeño en situaciones específicas,

Esta línea de pensamiento es corroborada por las investigaciones de González (2002) que define la competencia como una configuración psicológica. Otros autores concuerdan con lo anterior, destacando definiciones que señalan los recursos personales para la actuación profesional como la autonomía, flexibilidad, colaboración y responsabilidad, tales como 
LeBoterf (2001), al señalar que la competencia es equivalente a saber actuar e incluye: saber escoger, tomar iniciativas, arbitrar, correr riesgos, reaccionar ante lo imprevisto, contrastar, tomar responsabilidades y saber innovar.

Tomando los criterios de (Colunga 2006), la competencia debe preparar al hombre para la vida y hacerlo apto para afrontar situaciones profesionales nuevas, desconocidas, ambiguas, confusas, inesperadas, que requieren de urgente solución por su parte. Dicha autora destaca como elementos importantes a considerar: la elaboración, dominio y aplicación de estrategias individuales y grupales para mejorar las habilidades de comunicación; saber negociar; generar alternativas para resolver problemas. Asimismo significa la autoestima, la toma de decisiones, la solución de problemas, la jerarquización de tareas y motivos, vinculados a la comunicación interpersonal.

De forma general, los autores del presente artículo consideran que, en las mencionadas definiciones, se asume la competencia solamente como la combinación integrada de conocimientos, habilidades y actitudes conducentes a un desempeño adecuado y oportuno en diversos contextos, no teniendo en cuenta lo social, donde se ponen de manifiesto otros componentes de la conducta del individuo.

Los autores concuerdan con la manera de concebir las competencias y su trascendencia para la vida, a plenitud y convivencia humana, coincidiendo con el criterio de Tobón (2002) que propone conceptualizarlas como procesos complejos que las personas ponen en acciónactuación-creación, para realizar actividades sistémicas y resolver problemas docentes y de la vida cotidiana, con el fin de avanzar en la autorrealización personal, integrando el saber hacer (aplicar procedimientos y estrategias) con el saber conocer (comprender el contexto) y el saber ser (tener creatividad y motivación), teniendo los requerimientos específicos del contexto en continuo cambio, con autonomía intelectual, conciencia crítica, flexibilidad y espíritu de reto.

Además, es frecuente que en la literatura se manifieste una concepción limitada de las competencias, circunscritas a una habilidad, destreza o capacidad, como equivalente a una de estas, aprobando los componentes de habilidades en detrimento de los personales, los que abarcan emociones, valores, motivaciones, sentimientos, intereses, necesidades individuales y colectivas necesarias para el desarrollo humano, en correspondencia con el encargo social. Por lo tanto, se hace necesario pasar de las competencias como "un saber en contexto" a procesos de desempeño autocríticos y críticos de la realidad personal y del contexto externo, para ejercer una transformación.

Aquí se integran aspectos estructurales y funcionales inherentes a las competencias, porque no solo se enfatiza en sus atributos constitutivos y en su papel para el desempeño en situaciones disímiles. La complejidad de su naturaleza, el carácter multidimensional y su 
expresión en las actuaciones del sujeto, con frecuencia las profesionales, son peculiaridades atribuibles a los componentes funcionales de las competencias.

Desde la perspectiva puede decirse que su potencial regulador es notorio, su formación exige la integración en su estructura y funcionamiento de elementos de orden cognitivo y motivacional, donde ser competente implica disponer de recursos personológico que le permiten al sujeto funcionar con flexibilidad, reflexión personalizada, iniciativa, perseverancia, autonomía y perspectiva futura en su actuación que abarca la esfera moral que trasciende a la actuación.

Un proceso de formación de competencias profesionales que conciba sus componentes estructurales y funcionales que dinamice el desempeño y modo de actuación requiere enfatizar en la competencia de atomía emocional y moral como unidad dialéctica, en tanto la responsabilidad social y profesional está unida a un modo de actuación autónomo, lo cual es esencial en el caso de los estudiantes de la carrera de Turismo, a fin de responder a su encargo social.

\section{La competencia de autonomía emocional y moral.}

Desde el punto de vista etimológico, autonomía procede de "auto" que significa "por sí mismo" y "nomia (nomos)", que significa sistematización de las leyes, normas o conocimientos de una materia específica. Martínez (2006), en el análisis del término, significa la capacidad de tomar decisiones sin ayuda de otro.

Todas las emociones son, en esencia, impulsos que nos llevan a actuar, programas de reacción automática con los que nos ha dotado la evolución. La misma raíz etimológica de la palabra emoción proviene del verbo latino moveré (que significa moverse) más el prefijo "e-“", significando algo asi como "movimiento hacia" y sugiriendo, de ese modo, que toda emoción hay implícita una tendencia a la acción.

La moral (del gen. latín mōris, 'costumbre', y de ahí mōrālis, 'lo relativo a los usos y las costumbres') es un conjunto de normas, creencias, valores y costumbres que dirigen o guían la conducta de las personas en la sociedad. La moral permite distinguir qué acciones son correctas (buenas) y cuáles son incorrectas (malas). Otra perspectiva la define como el


social.

Analizando la etimología de estos términos, se puede aseverar que las normas, creencias, valores y costumbres, dirigen la conducta del individuo, llevándolo actuar y a propiciar el desarrollo de la capacidad de tomar decisiones sin la ayuda de otros.

Múltiples autores (Martin, 2004) (Martínez, 2009a y 2013b) refieren a la competencia de autonomía, como la capacidad de elegir con criterio propio, de llevar a cabo proyectos y 
acciones necesarias para desarrollar las opciones y planes en el ámbito personal, social y laboral.

Martínez (2009a y 2013b) considera las competencias emocionales como el nivel de funcionamiento de la personalidad que se constituye en el recurso personal del estudiante, le permite cumplir las tareas con independencia, responsabilidad y efectividad ante la toma de decisiones, a partir del planteamiento consciente de objetivos, la planificación de acciones y la defensa del juicio argumentado, auto valorando las posibilidades y recursos que posee para el futuro desempeño con compromiso social.

Las competencias emocionales o socio-afectivas, comúnmente se asocian al término de inteligencia emocional (Goleman, 1996). Asimismo, Goleman (2002) comprende las competencias emocionales: como autoconciencia, autorregulación, motivación, empatía y destrezas sociales, las que pueden ser enseñadas.

Las competencias emocionales permiten el reconocimiento, manejo de los sentimientos y las emociones, tanto propias como ajenas, para lograr relaciones mutuamente satisfactorias entre el hombre y su medio laboral y social. Según Bisquerra (2009), las competencias emocionales son el conjunto de conocimientos, capacidades, habilidades y actitudes necesarias para tomar conciencia, comprender, expresar y regular de forma apropiada los fenómenos emocionales. La finalidad de estas competencias se orienta a promover el bienestar personal y social.

Enfatiza en el vínculo que González (1982) aprecia entre autonomía y esfera moral al señalar que la autonomía moral se expresa en la edad escolar superior, que con relativa independencia moral y social el estudiante orienta su vocación, valora las posibilidades prácticas futuras e internas, apoyado en el ideal, concepción del mundo y elaboración personal mediante juicios, experiencias y vivencias que se convierten en motivo moral y tendencias motivacionales, se plantea aspiraciones, se orienta en la vida e incorporación laboral, asume responsabilidad y compromiso social que regulan su vida, lo que adquiere en su desarrollo y por la educación.

La autonomía emocional y moral, vista desde la responsabilidad, la expresión motivacional, las aspiraciones e intenciones profesionales, la toma de decisión efectiva y el compromiso social, se apreciaron en estudios precedentes (Martínez, 2009, 2013), elementos que se ratifican en el estudio de la autonomía y el compromiso social responsable, donde se revelaron indicadores como la toma de decisiones con responsabilidad, la implicación afectiva y satisfacción en el cumplimiento responsable de las normas y exigencias del desempeño profesional, el autocontrol moral y emocional, resultados de interés para este estudio.

Los autores del presente artículo coinciden en concebir la autonomía emocional como un concepto amplio que incluye un conjunto de características y elementos relacionados con la autogestión personal, entre las que se encuentran la automotivación, la actitud positiva ante 
la vida, responsabilidad y la capacidad para analizar críticamente las normas sociales, desde una posición ética y en consonancia con las normas morales que siempre han afectado al ser humano.

Ya que las normas morales existen en la conciencia de cada uno, esto provoca que existan diferentes puntos de vista y por ende problemas en el momento de considerar las diferentes respuestas que ejercen las personas frente a ellas, debido al pluralismo que existe en las tendencias frente a un mismo acto, esto es que, para cuando algunas personas un acto es lo correcto, para otros es inmoral. Esto está íntimamente ligado con el tema de los valores ya que normalmente se dice que lo que se hace por obligación, pierde todo mérito, en cambio, cuando se realiza por propio convencimiento, adquiere valor moral. Con esto se da a entender que la obligación moral le quita al hombre la única posibilidad de ser el mismo, de acuerdo con su propia moralidad y con su propio criterio.

El estudio teórico realizado ha permitido definir las estrechas relaciones que se pueden observar entre la autonomía emocional y moral de la persona y estas se expresan en que:

- Las emociones dan la oportunidad de aumentar el grado de conocimiento que se tiene a cerca de la propia moralidad.

- Las emociones tienen un fuerte impacto motivacional a la hora de rectificar un comportamiento moral inadecuado, reforzar lo que es correcto y reparar el mal causado.

- Las emociones mejoran la percepción moral, orientan y proporcionan apoyo a los principios morales.

- Las emociones no sólo afectan a lo que una persona ve o a cómo lo ve, sino que también influyen en como los demás perciben y responden a esa persona.

- Las emociones son una fuente de información fundamental a la hora de tomar decisiones, ya que constituyen una guía básica para la conducta moral.

De esta manera se evidencia una unión indisoluble entre lo emocional y lo moral, pues coincidiendo con Iriarte et. al.. (2006), las emociones conscientes, educadas y bien gestionadas, ayudan a convertir al estudiante en un sujeto maduro y responsable, promueven el reconocimiento de los propios valores, el compromiso, la confianza, la integridad y las conductas de cooperación.

El desarrollo de la autonomía moral y emocional de los profesionales en formación de la Carrera de Turismo, es una necesidad y demanda planteada desde su misión, orientada a formar profesionales competentes, comprometidos, responsables socialmente, dignos, con principios éticos y espíritu de sacrificio, que les permita transformar el entorno social, mediante el desarrollo de un turismo de alto nivel de satisfacción al cliente como gestores de uno de los renglones más importantes del país.

La necesidad de garantizar un profesional de turismo comprometido y responsable con las políticas y lineamientos del estado cubano, la apertura y estrategias de desarrollo del sector 
privado, los nuevos perfiles que imponen los avances tecnológicos y las tendencias internacionales aplicables a Cuba, demandan egresados con competencias que potencien la actividad turística, a partir de una formación que abarque en ellos la competencia de autonomía moral y emocional y les permita enfrentar con efectividad los desafíos presentes y futuros en su ámbito de acción, aporte social que constituye un reto importante de esta investigación, pues la incorporación y desarrollo de la competencia de autonomía moral y emocional responde y tributa a la carencia y demanda reveladas en la dinámica del proceso de formación del profesional del Turismo y en la práctica profesional.

\section{Estado actual de la competencia de autonomía emocional y moral en la Facultad de Turismo en la Universidad de La Habana.}

Los factores sociales y emocionales están presentes incuestionablemente en el escenario educativo, por lo que en la formación profesional de los estudiantes de la carrera de Turismo se debe tomar esto en consideración y asumir el compromiso para desarrollaren las nuevas generaciones la capacidad de afrontar de forma creativa y flexible las situaciones, problemas e inconvenientes que puedan surgir en el mundo tanto académico como laboral.

El desarrollo de estas competencias reviste especial importancia para el Licenciado en Turismo ya que una persona autónoma supone aprender a desenvolverse a nivel personal, profesional y social en un mundo cada vez más complejo, va a fortalecer su trabajo, su relación con agentes y clientes tanto nacionales como extranjeros. Además, actualmente la competencia de autonomía emocional y moral se considera un aspecto importante de las habilidades de empleabilidad. En el mundo laboral se acepta que la productividad depende de una fuerza de trabajo que sea emocionalmente competente (Elias, 1999).

Es necesario señalar, que en la actualidad cubana las empresas demandan asiduamente profesionales comprometidos con su entorno, su organización y su profesión, con elevado nivel de creatividad, versatilidad, autonomía, adaptabilidad y comprometidos con su entorno, su organización y su profesión. Por ello, se torna imperativo abrir paso a nuevas metodologías didácticas que tributen a la formación de profesionales autónomos, responsables ante la toma de decisiones, que desarrollen aptitudes para el análisis crítico y constructivo, y el trabajo en equipo; elementos indispensables para el buen resultado del servicio que se ofrece y para la contribución colectiva a la solución de las nuevas situaciones que se presentan.

En la Facultad de Turismo a pesar de habérsele dado tratamiento a las competencias profesionales a través de trabajos como: "Propuesta de perfil del cargo por competencias laborales del recepcionista hotelero de la villa turística Horizontes Soroa” (Maestres, 2012), "Diagnóstico de competencias organizacionales en el Hotel Inglaterra" (Rodríguez, 2012) y "Evaluación de las competencias profesionales de los Licenciados en Turismo ante los nuevos desafíos en su ámbito de acción" (Ascón, 2013); no se hace referencia a la 
competencia autonomía emocional; aun cuando los resultados de los trabajos mencionados anteriormente tributan a la necesidad de desarrollarla también en la carrera.

En estos estudios se plantea que los profesionales del turismo deben responder a las demandas del sector, entre ellas el desarrollo del trabajo en equipo, la toma de decisiones acertadas y oportunas, la habilidad para las relaciones interpersonales, la auto-superación continua en los avances de la profesión, traer el conocimiento a la práctica y la creatividad en la labor profesional. Estos constituyen elementos indispensables para el buen resultado del servicio que se ofrece y para la contribución colectiva a la solución de las nuevas situaciones.

Utilizando métodos y técnicas como la observación, análisis documental, encuestas a estudiantes y profesores así como soportes estadísticos para tabular datos, se logra realizar un diagnóstico del comportamiento actual de la competencia de autonomía emocional y moral de los estudiantes de la Facultad de Turismo.

Donde se destaca que la asignatura de Comunicación Interpersonal, es la única cuyos contenidos guardan relación con la autonomía emocional y moral, fomentando el contacto con los públicos internos, externos, el trabajo en equipo a través de la formación de habilidades para la comunicación. Sin embargo, esta asignatura solo se imparte en 1er año y no trabaja otras dimensiones de la autonomía emocional y moral. Por ello es necesario enseñarles a los estudiantes como dirigir las emociones tanto para su formación, como para enfrentar los nuevos cambios que se avecinan en el sector. Sería conveniente que los objetivos educativos de varias asignaturas de la carrera buscaran desarrollar esta competencia.

Otra de las razones es que la facultad cuenta con un claustro de profesores que trabaja para elevar la efectividad de la labor docente educativa, logrando un ambiente que sea reflejo de altos valores en la comunidad universitaria y de hábitos de educación formal.

Si bien la competencia autonomía emocional y moral no ha sido tratada de manera explícita en la facultad, los resultados de las encuestas aplicadas a los docentes demuestran que poseen un dominio del tema. Entre los criterios abordados los más reiterativos hacen alusión al conjunto de conocimientos, actitudes, valores y habilidades que garantizan en el sujeto su adaptación y regulación conductual ante los cambios en las situaciones del entorno, a la capacidad para asumir procesos de autorregulación y control en el desempeño personal y profesional del sector. La búsqueda de soluciones de manera eficaz y creativa a los problemas y el desempeño exitoso, evitando que estados emocionales negativos afecten las metas a cumplir.

El análisis de los resultados permite decir que los estudiantes presentan dificultades en la responsabilidad, automotivación, análisis crítico, actitud positiva y autocontrol. En el caso del manejo de relaciones solamente se ve afectado en tercer año. Las irregularidades se pueden resumir en el cumplimiento del reglamento docente disciplinario, asumir la 
responsabilidad por las decisiones tomadas, implicarse emocionalmente al emprender acciones sistemáticas, afrontar de forma crítica y autocrítica las situaciones negativas de aprendizaje, el reconocimiento de los errores propios, mantener una actitud positiva ante el incremento de las exigencias académicas, la perseverancia para enfrentar los obstáculos o decepciones en la vida estudiantil y la búsqueda de soluciones a los problemas de manera entusiasta y creativa. Además, la flexibilidad para afrontar los cambios y las nuevas situaciones de aprendizaje, el manejo de conflictos y el control de las emociones y los impulsos. Dichos resultados se resumen en la tabla1.

Tabla 1: Insuficiencias constatadas en la exploración sobre la competencia de autonomía emocional y moral.

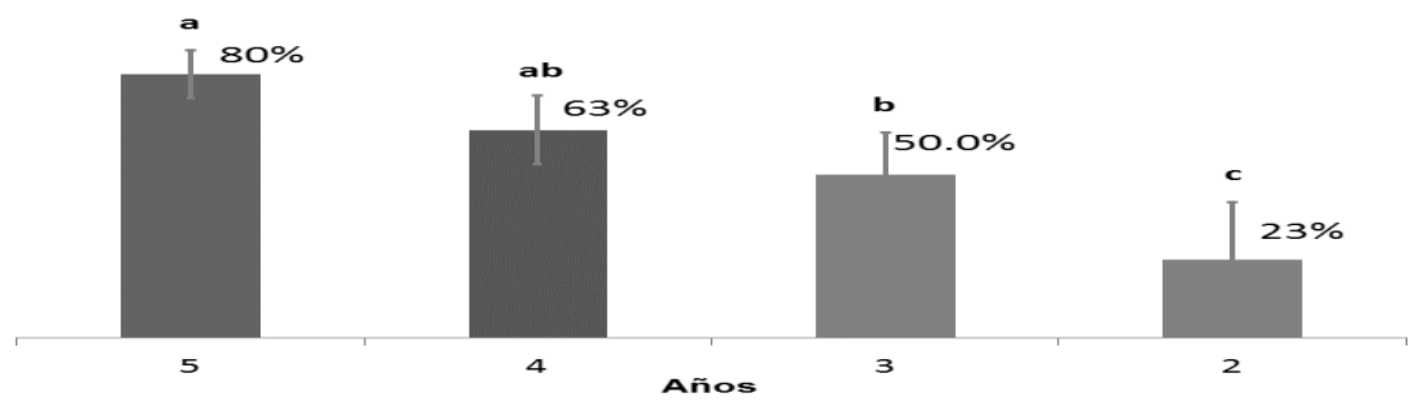

Fuente: Elaboración propia.

\section{Deficiencias encontradas:}

- Dificultades en asumir la responsabilidad por las decisiones tomadas.

- Problemas para implicarse emocional y moralmente al emprender acciones sistemáticas que contribuyan a su desarrollo integral con resultados satisfactorios.

- No se muestran flexibles para afrontar los cambios y nuevas situaciones de aprendizaje.

- Limitado autocontrol y manejo de conflictos, de las relaciones y el trabajo en equipo.

- Limitaciones en la comunicación interpersonal.

- El análisis crítico ante situaciones determinadas es poco importante para su formación.

- Limitada búsqueda de alternativas de solución a los problemas de manera creativa.

\section{Conclusiones.}

- El análisis conceptual y evolución de la competencia de autonomía emocional y moral demostró la relación dialéctica que existe entre las mismas.

- La competencia de autonomía emocional y moral tiene un alcance amplio que incluye un conjunto de características y elementos relacionados con la autogestión personal, entre las que se encuentran la automotivación, la actitud positiva ante la vida, responsabilidad y la capacidad para analizar críticamente las normas sociales, desde una 
posición ética y en consonancia con las normas morales que siempre han afectado al ser humano.

- El uso de diferentes técnicas y métodos permitió realizar un diagnóstico del comportamiento actual de la competencia de autonomía emocional y moral de los estudiantes de la Facultad de Turismo.

\section{Referencias bibliográficas.}

Ascón Villa, J.E. Evaluación de las competencias profesionales de los Licenciados en Turismo ante los nuevos desafíos en su ámbito de acción. Trabajo de diploma. Universidad de La Habana. 2013.

Bisquerra, A. R. (2004). Competencias emocionales y educación emocional. IV Jornadas Técnicas de Orientación Profesional. Consejo Aragonés de Formación Profesional Zaragoza. 23 y 24 de noviembre.

Bisquerra, A. R (2009). Psicopedagogía de las emociones. Madrid.

Colunga, S. y García, J. (2006). Reflexiones acerca de la noción de competencia.

Elias, M.J (1999). Educación con Inteligencia Emocional. Editorial Plaza \& Janés. México.

García, J.A. y Tobón, S. (2006).Competencias, calidad y educación superior. Bogotá: Magisterio.

Gonczi, A. y Athanasau, J. (1996). Instrumentación de la educación basada en competencias. Perspectiva de la teoría y la práctica en Australia. Editorial Limusa.

González, V. (2002). ¿Qué significa ser un profesional competente? Reflexiones desde una perspectiva psicológica. Revista Cubana de Educación Superior, 12 (1) 45-53.

Goleman, D (1996). La inteligencia emocional en la empresa. Buenos Aires: Javier Vergara Editor.

Goleman, D (2002). Competencia Emocional. La iniciativa de comunicación. Disponible en: http://www.la red de cila.com.

González, B. (2016). La categoría formación. En: Visión pedagógica de la formación universitaria actual. Ortiz, T. y Sanz, T. (coord.), Editorial UH, La Habana, 296p.

Hué, C (2013). Una experiencia de formación en competencias emocionales del profesorado universitario. Universidad de Zaragoza, 150p.

Le Boterf, G. (2001). Ingeniería de las competencias. Barcelona: Epise.

Maestres, Y (2012). Propuesta de perfil del cargo por competencias laborales del recepcionista hotelero de la villa turística Horizontes Soroa. Trabajo de diploma. Universidad de La Habana. 
Martín Fernández, R. La Formación en el Turismo de Nivel Superior. Revista. Apuntes. 2004.

Martínez, O. (2009). Ideas acerca de investigación sobre Responsabilidad Social Universitaria (RSU). Material inédito digitalizado. CEPES.

Martínez, O. (2013). Análisis evolutivo de la autonomía. Material inédito digitalizado. CEPES.

Martínez, O. Ideas acerca de investigación sobre Responsabilidad Social. Universitaria (RSU). Material inédito digitalizado. CEPES.2009a, 2013b.

MES (2007). Reglamento de Trabajo Docente Metodológico del Ministerio de Educación Superior.

OEI (2010). Declaración de Mar del Plata. XX Cumbre Iberoamericana de Jefes de Estado y de Gobierno. Mar del Plata, Argentina, 3 y 4 de diciembre de 2010. Disponible en http://segib.org/wpcontent/uploads/DCL-MAR-DEL-PLATA-JEGXX-E.pdf.

Pinto, L. (1999). La escuela como contexto de enseñanza-aprendizaje.

Rodríguez, A (2012). Diagnóstico de competencias organizacionales en el Hotel Inglaterra. Trabajo de diploma. Universidad de La Habana.

Tobón, S. (2002). Las competencias en el sistema educativo: de la simplicidad a la complejidad. Colombia.

Tobón, S. (2005). Formación basada en competencias. Pensamiento complejo, diseño curricular y didáctica.

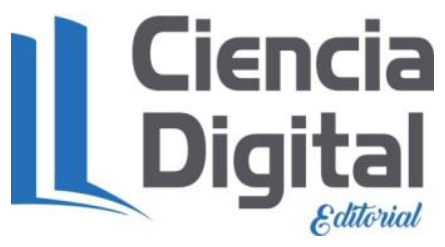




\section{Para citar el artículo indexado.}

Valdés Y. \& Vázquez Y. . (2018). Formación de la competencia de autonomía emocional y moral en estudiantes de turismo. Revista electrónica Ciencia Digital 2(3), 203-215. Recuperado desde:

http://cienciadigital.org/revistacienciadigital2/index.php/CienciaDigital/article/view/147/13 $\underline{2}$

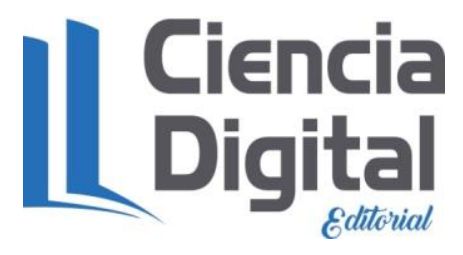

El artículo que se publica es de exclusiva responsabilidad de los autores y no necesariamente reflejan el pensamiento de la Revista Ciencia Digital.

El articulo queda en propiedad de la revista y, por tanto, su publicación parcial y/o total en otro medio tiene que ser autorizado por el director de la Revista Ciencia Digital.
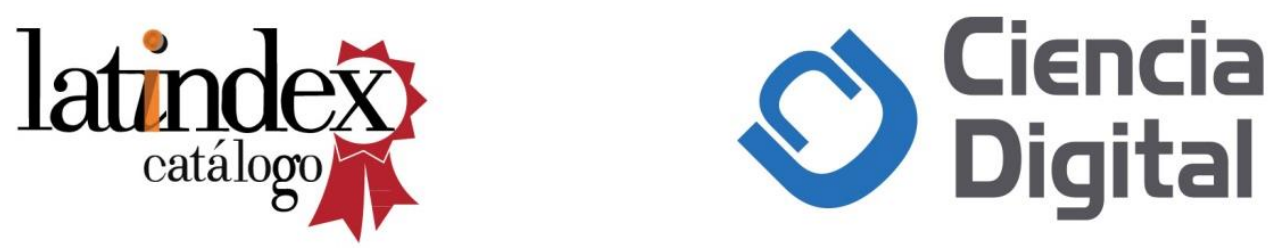